\title{
ANATOMÍA DE LA MADERA DE OCHO ESPECIES DE ANGIOSPERMAS
}

\author{
DE CLIMA TEMPLADO
}

\author{
Alejandra Quintanar, Carmen de la Paz Pérez Olvera, \\ Isabel de la Cruz laina y Daría Razo Balcazar
}

\begin{abstract}
Departamento de Biología, División de Ciencias Biológicas y de la Salud. Universidad Autónoma MetropolitanaIztapalapa. Apdo. Postal 55-535, Delegación Iztapalapa, 09340 México, D.F., México.
\end{abstract}

\begin{abstract}
Resumen. Se presentan los resultados del estudio anatómico de la madera de Alnus acuminata Kunth, Ostrya virginiana Rose, Quercus dysophylla Benth., Quercus glabrescens Benth., Arbutus glandulosa DC., Arbutus tessellata Sorensen, Styrax argenteus Presl. y Buddleia wrightii Robinson, de muestras provenientes de los estados de Jalisco y Puebla. Para cada taxa estudiado, se describen los caracteres anatómicos de los vasos, parénquima axial, radios y fibras; también se sugieren algunos usos para la madera de estas especies.

Palabras clave: anatomía, Jalisco, maderas templadas, Puebla.

Abstract. Wood anatomy of Alnus acuminata Kunth, Ostrya virginiana Rose, Quercus dyseophylla Benth. and Quercus glabrescens Benth., Arbutus glandulosa DC., Arbutus tessellata Sorensen, Styrax argenteus Presl. and Buddleia wrightii Robinson, collected in the state of Jalisco and Puebla are given. For each studied taxa, distinctive anatomical features are rebounded, and for each microscopic characteristic, photographs are given. Recommendations on their uses are suggested.
\end{abstract}

Key words: anatomy, Jalisco, Puebla, temperate woods.

E $\mathrm{n}$ los bosques de pino-encino como en los mesófilos de montaña de México, crecen especies maderables que representan un gran potencial económico. En los primeros, además de Pinus y Quercus, son comunes los géneros Alnus, Arbutus y Buddleia, que presentan fustes rectos con diámetros de hasta $30 \mathrm{~cm}$ y alturas de 9 a $11 \mathrm{~m}$. En estos ecosistemas el género Quercus es el más abundante de las latifoliadas con una gran diversidad de especies (Zavala, 1990), que son destinadas para elaborar carbón (SARH, 1989).

Rzedowski (1992) menciona que los bosques mesófilos de montaña presentan la mayor diversidad de especies ya que existen aproximadamente 90 géneros y 300 especies arbóreas, entre las que se encuentran Ostrya y Styrax que presentan madera de excelente calidad (de la Paz Pérez y Corral, 1980; de la Paz Pérez et al., 1982; Rzedowski, 1988). Sin embargo, el uso tradicional al que se han destinado es principalmente para artesanía (Guridi, 1980).

Estos ecosistemas han sido devastados pues la mayoría de sus especies son taladas injustificadamente o sufren la acción del arrastre que se hace de la madera de pino cuando es extraída, lo que ha ocasionado el deterioro de grandes áreas boscosas (Reyes, 1994; Dirzo, 1995).

En México las especies latifoliadas de clima templado representan una fuente de riqueza poco conocida desde el punto de vista maderable y sólo se ha estudiado la anatomía de unas cuantas. De las diez especies de Alnus (Standley y Steyermark, 1952), sólo se han descrito A. firmifolia (Rebollar, 1977), A. jorullensis (Barajas, 1980; de la Paz Pérez y Quintanar, 1994) y A. arguta (de la Paz Pérez y Corral, 1980). De las dos especies de Ostrya (Standley, 1961), sólo se ha descrito O. guatemalensis (de la Paz Pérez et al., 1982). De las 200 especies de Quercus (Zavala, 1990) se han estudiado 30 (de la Paz Pérez, 1974, 1976, 1982, 1985; Corral, 1981; Ortega et al., s/f). De las diez especies de Arbutus, de las ocho de Styrax y de las 20 de Buddleia que menciona Standley (1924), sólo se han descrito A. xalapensis, $S$. ramirezii (de la Paz Pérez y Corral, 1980) y B. parviflora (de la Paz Pérez et al., 1980). 


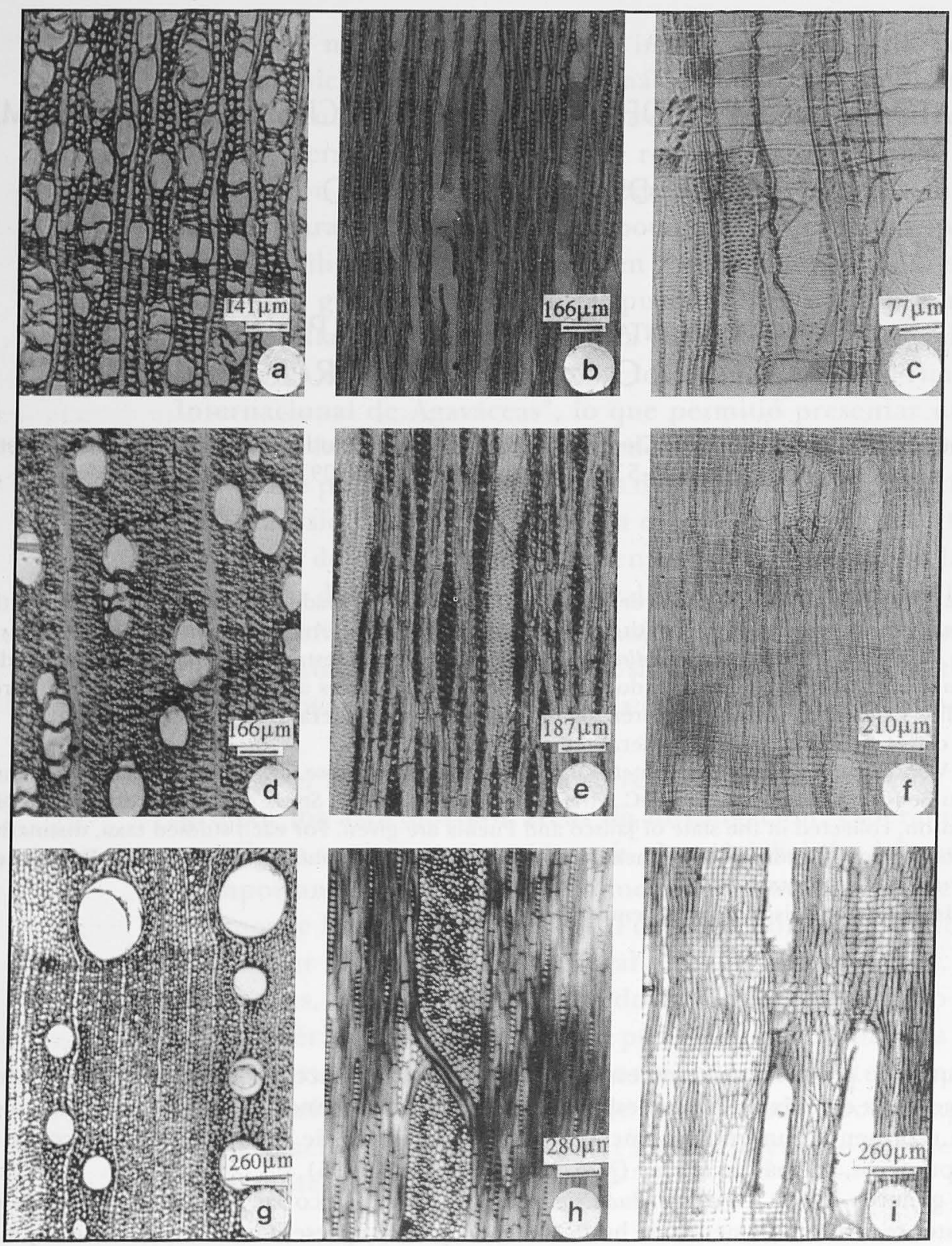

Lámina 1. Cortes transversales (izquierda), cortes tangenciales (centro) y cortes radiales (derecha); figuras a-c Alnus acuminata; d-f Ostrya virginiana; g-i Quercus dysophylla.

Los taxa estudiados en este trabajo no han sido descritos anatómicamente, por lo que el estudio de su madera amplía el conocimiento de estas especies.

Los estudios anatómicos aparte de contribuir al conocimiento de las especies, aportan información básica para el mejor aprovechamiento de la madera (Jane, 1970; Hoadley, 1989), ya que con ellos, es posible plantear algunos criterios para promover su introducción al mercado nacional.

\section{Materiales y métodos}

En el estado de Jalisco, en un bosque de pino-encino, se recolectaron Arbutus tessellata Sorensen (Ericaceae) y Buddleia wrightii Robinson (Loganiaceae) y en un bosque mesófilo de montaña, Ostrya virginiana Rose (Betulaceae) y Styrax argenteus Presl. (Styracaceae). En el estado de Puebla, en un bosque de pino-encino, se muestrearon Alnus acuminata Kunth (Betulaceae), 
Quercus dysophylla Benth. (Fagaceae, Erythrobalanus), Q. glabrescens Benth. (Fagaceae, Leucobalanus) y Arbutus glandulosa DC. (Ericaceae).

Se muestreó un árbol para cada especie y los ejemplares de respaldo se depositaron en el Herbario Metropolitano (UAMIZ). De cada árbol se obtuvo una troza de $1.30 \mathrm{~m}$ de largo, medida de la base del tronco hacia la copa.

De la parte superior de cada troza, se cortaron dos rodajas de $2 \mathrm{~cm}$ de grosor de las que se sacaron cubos de $2 \mathrm{~cm}$ con los que se hicieron cortes transversales, tangenciales y radiales, que se tiñeron con verde iodo, así como material disociado, para elaborar preparaciones fijas que se utilizaron para hacer el estudio microscópico. Los cubos se ablandaron previamente siguiendo las técnicas de Koller (1927) y Franklin (1946). El tejido se disoció usando el método de Jeffrey (Johansen, 1940) y se tiñó con pardo de Bismarck.

Del resto de la troza se obtuvieron tablillas de $15 \times 7 \times 1 \mathrm{~cm}$ con las que se determinaron las características macroscópicas, de acuerdo con Tortorelli (1956); para el color se usaron las tablas de Munsell (1990).

Las descripciones microscópicas se hicieron como se indica: los anillos de crecimiento, la porosidad, el arreglo de poros y su contorno, así como el tipo de parénquima axial se describieron en cortes transversales. Las punteaduras intervasculares y el tipo de radios en cortes tangenciales; la placa perforada, las paredes terminales de los elementos vasculares, los tipos celulares que forman los radios, así como las punteaduras vaso-radio se hicieron en cortes radiales, siguiendo algunas de las indicaciones del Comité de la Asociación Internacional de Anatomistas de la Madera (IAWA) de 1989. Por otro lado en los cortes transversales se cuantificó y midió el número de vasos $/ \mathrm{mm}^{2}$ y el diámetro tangencial de los mismos; en los tangenciales se cuantificó el número de radios/ mm y se midió la altura de los uniseriados así como la anchura y número de series de los multiseriados. En las tablillas tangenciales de Quercus se midieron la altura y número de radios de los multiseriados y en las tangenciales de Alnus los agregados. En material disociado se midieron la longitud de los elementos de vaso y fibras y el grosor de la pared de éstas. Se hicieron entre 100 y 300 mediciones dependiendo del carácter. Los datos obtenidos, se sometieron a un análisis estadístico univariado con un error de muestreo del $5 \%$. Cada carácter se categorizó utilizando el valor de la media de acuerdo con las denominaciones sugeridas por Chattaway (1932) y los Comités de la IAWA de 1937 y 1939. Para cada carácter se dan los valores de la media y entre paréntesis los valores mínimo y máximo. Se ilustran en las láminas 1-3 los caracteres anatómicos distintivos de las especies estudiadas.
Los taxa se arreglaron de acuerdo al sistema de clasificación de Engler modificada por Dalla Torre y Harms (1963).

\section{Resultados}

\section{Alnus acuminata (lámina 1 , figuras $a-c$ )}

Características macroscópicas. La madera no presenta diferencia de color entre albura y duramen, es de color amarillo rojizo (7.5YR 7/6); no tiene olor ni sabor característicos, el brillo es mediano, el veteado es liso, la textura es fina y el hilo es recto. Los elementos constitutivos de la madera son visibles a simple vista, los anillos de crecimiento están marcados.

Características microscópicas. Los anillos de crecimiento están marcados por 6 (5-10) hileras de fibras radialmente comprimidas. La madera presenta porosidad difusa, los vasos están arreglados en hileras radiales, son numerosos, de $75(50-100) / \mathrm{mm}^{2}$, la mayoría son múltiples radiales de 2 a 8 y $4 \%$ son solitarios, de contorno angular, el diámetro tangencial es moderadamente pequeño, de $50(40-60) \mu \mathrm{m}$. Los elementos de vaso son de longitud mediana, de 530 (316-750) $\mu \mathrm{m}$, con placas perforadas escalariformes, de 15 (9-20) barras, de paredes terminales oblicuas y punteaduras areoladas opuestas de forma angular. Las punteaduras de vaso-radio son redondeadas.

El parénquima axial es apotraqueal difuso, formado por cordones de 5 a 8 células.

Los radios son de una a tres series, la mayoría uniseriados, algunos agregados, homocelulares, formados por células procumbentes; son muy numerosos, de $16(10-22) / \mathrm{mm}$, extremadamente bajos, de 360 (120-810) $\mu \mathrm{m}$ y de anchura mediana, de 50 (30-70) $\mu \mathrm{m}$. Los agregados son altos, mayor a $1 \mathrm{~mm}$.

Las fibras son de tipo libriforme, moderadamente cortas, de 815 (513-1303) $\mu \mathrm{m}$, diámetro mediano, de 23 (11-33) $\mu \mathrm{m}$, paredes delgadas, de 2 (1-3) $\mu \mathrm{m}$ y con punteaduras simples en las caras radiales.

\section{Ostrya virginiana (lámina 1 , figuras $d-f$ y lámina 3 , figura $k$ )}

Características macroscópicas. La madera presenta diferencia de color entre albura y duramen, la albura es rosa (5YR 7/4) y el duramen castaño rojizo (5YR 6/ $3)$; no tiene olor ni sabor característicos, el brillo es mediano, el veteado es liso, la textura es fina y el hilo recto. Los elementos constitutivos de la madera son poco visibles a simple vista, los anillos de crecimiento están poco marcados.

Características microscópicas. Los anillos de crecimiento están marcados por 4 (3-7) hileras de fibras radial- 


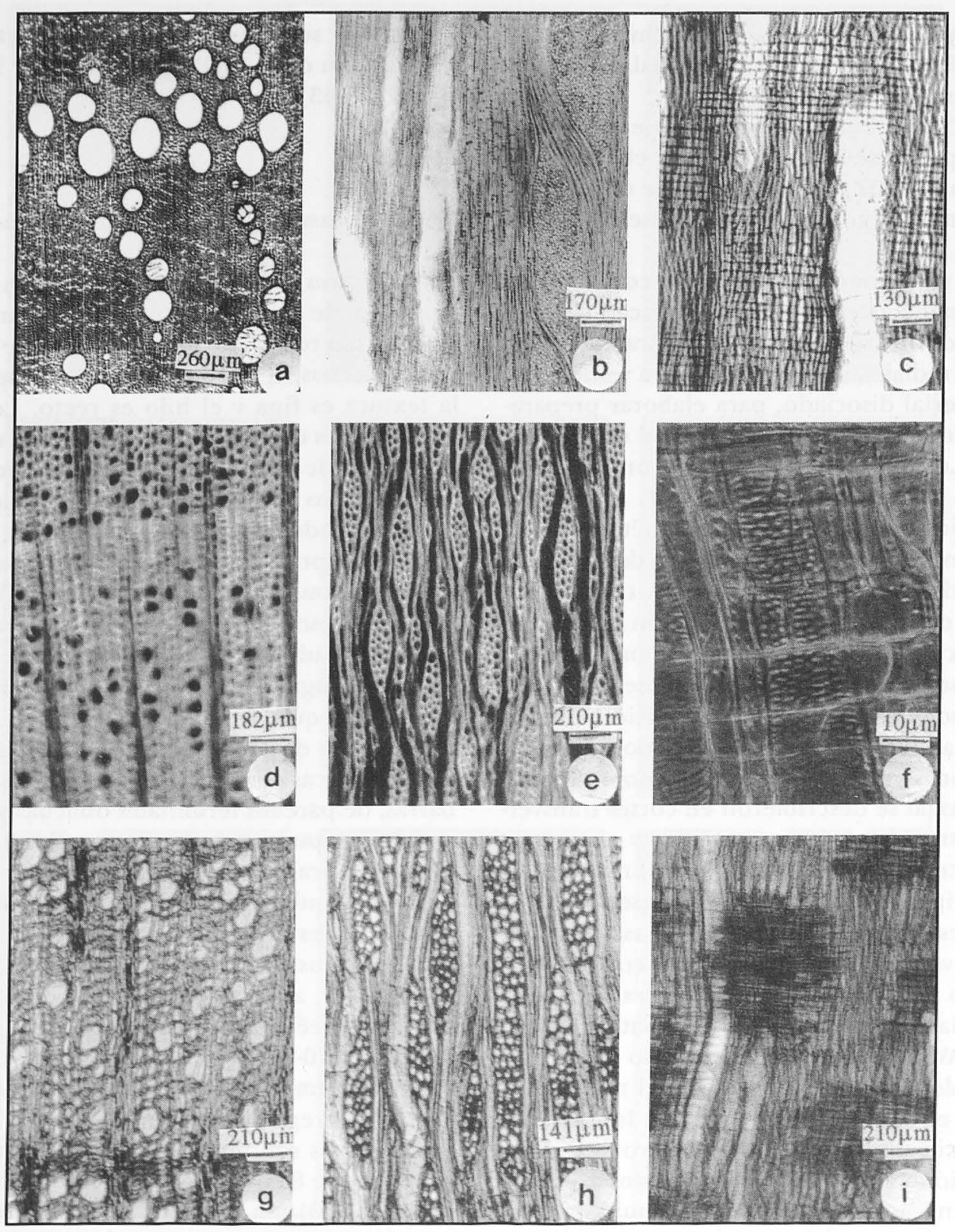

Lámina 2. Cortes transversales (izquierda), cortes tangenciales (centro) y cortes radiales (derecha); figuras $a-c$ Quercus glabrescens; d-f Arbutus glandulosa; $g$-i Arbutus tessellata.

mente comprimidas. La madera presenta porosidad difusa, los vasos están arreglados en hileras radiales, son numerosos, de $30(19-51) / \mathrm{mm}^{2}$, la mayoría son múltiples radiales de 2 a 7 y $6 \%$ son solitarios, de contorno angular, el diámetro tangencial es moderadamente pequeño, de 75 (54-129) $\mu \mathrm{m}$. Los elementos de vaso son de longitud mediana, de 690 (400-800) $\mu \mathrm{m}$, con placas perforadas simples, de paredes terminales oblicuas y punteaduras areoladas alternas de forma poligonal; presentan engrosamientos helicoi- dales. Las punteaduras vaso-radio son redondeadas.

El parénquima axial es apotraqueal difuso, formado por cordones de 4 a 6 células.

Los radios son de una a tres series, la mayoría biseriados, homocelulares, formados por células procumbentes; son muy numerosos, de 11 (7-14)/mm, extremadamente bajos, de $389(160-800) \mu \mathrm{m}$ y moderadamente finos, de $34(20-50) \mu \mathrm{m}$.

Las fibras son de tipo libriforme, medianas, de 1451 (1200-1600) $\mu \mathrm{m}$, diámetro mediano, de 19 (12-28) $\mu \mathrm{m}$, 
paredes gruesas, de $8(6-10) \cdot \mu$ m y con punteaduras simples en las caras radiales.

\section{Quercus dysophylla (lámina 1 , figuras $g-i$ )}

Características macroscópicas. La madera no presenta diferencia de color entre albura y duramen, es de color castaño pálido (10YR 6/3), los radios multiseriados son de color castaño (10YR 5/3) contrastando ligeramente con el resto de los elementos constitutivos de la madera; no tiene olor ni sabor característicos, el brillo es mediano, el veteado es suave, la textura es mediana y el hilo es recto. Los elementos constitutivos de la madera son visibles a simple vista, los anillos de crecimiento están marcados.

Características microscópicas. Los anillos de crecimiento están marcados por dos a tres hileras de vasos de madera temprana. La madera presenta porosidad anular, los vasos de la madera tardía, están arreglados en patrones radiales, son pocos, de $5(3-8) / \mathrm{mm}^{2}$, solitarios; de contorno oval y algunos circulares, el diámetro tangencial de los vasos de la madera temprana es grande, de $230(170-340) \mu \mathrm{m}$ y el de la tardía es mediano, de $110(70-150) \mu \mathrm{m}$. Los elementos de vaso son de longitud mediana, de 495 (316-711) $\mu \mathrm{m}$, con placas perforadas simples, de paredes terminales oblicuas y punteaduras areoladas alternas de forma oval. Las punteaduras de vaso-radio son simples, ovales y con arreglo vertical. Algunos vasos presentan escasas tílides. Traqueidas vasicéntricas se encuentran alrededor de algunos vasos de la madera temprana.

El parénquima axial es apotraqueal difuso en agregados, formado por cordones de 3 a 4 células.

Los radios son uniseriados y multiseriados, homocelulares, formados por células procumbentes; los primeros son muy numerosos, de $13(8-22) / \mathrm{mm}$ y extremadamente bajos, de 98 (126-300) $\mu \mathrm{m}$. Los multiseriados son poco numerosos, de $3(2-3) / 5 \mathrm{~mm}$, moderadamente altos, de $0.6(0.3-0.9) \mathrm{cm}$, anchos, de $250(119-474) \mu \mathrm{m}$ y de 12 a 28 series.

Las fibras son de tipo libriforme, medianas, de 1065 (687-1438) $\mu \mathrm{m}$, diámetro mediano, de 25 (16-36) $\mu \mathrm{m}$, paredes medianas, de 7 (5-12) $\mu$ m y con punteaduras simples en las caras radiales.

4. Quercus glabrescens (lámina 2, figuras $a-c$ y lámina 3 , figura $j$ )

Características macroscópicas. La madera no presenta diferencia de color entre albura y duramen, es de color blanco (10YR 8/2); no tiene olor ni sabor característicos, el brillo es mediano, el veteado es liso, la textura es fina y el hilo es recto. Los elementos constitutivos de la madera son visibles a simple vista, excepto los rayos, los anillos de crecimiento están bien marcados. Características microscópicas. Los anillos de crecimiento están marcados por dos hileras de vasos de madera temprana. La madera presenta porosidad anular, los vasos de la madera tardía están arreglados en patrones radiales, son pocos, de $7(4-12) / \mathrm{mm}^{2}$, solitarios, de contorno oval y algunos circulares, el diámetro tangencial de los vasos de la madera temprana es mediano, de 104 (83-151) $\mu \mathrm{m}$ y el de la tardía es muy pequeño, de 43 (29-61) $\mu \mathrm{m}$. Los elementos de vaso son de longitud mediana, de 474 (308-706) $\mu \mathrm{m}$, con placas perforadas simples, de paredes terminales oblicuas y punteaduras areoladas alternas de forma oval. Las punteaduras de vaso-radio son simples, ovales y con arreglo vertical. Algunos vasos presentan escasas tílides. Traqueidas vasicéntricas se encuentran alrededor de algunos vasos de la madera temprana.

El parénquima axial es difuso en agregados, formado por cordones de 3 a 4 células.

Los radios son uniseriados y multiseriados, homocelulares, formados por células procumbentes; los primeros son muy numerosos, de $15(10-20) / \mathrm{mm}$ y extremadamente bajos, de $132(90-224) \mu \mathrm{m}$. Los multiseriados son poco numerosos, de $3(2-3) / 5 \mathrm{~mm}$, altos, de $1.0(0.5-1.0) \mathrm{cm}$, moderadamente anchos, de $190(79-324) \mu \mathrm{m}$ y de 7 a 21 series.

Las fibras son de tipo libriforme, medianas, de 1178 (672-1790) $\mu \mathrm{m}$, diámetro mediano, de 24 (16-34) $\mu \mathrm{m}$, paredes medianas, de 7 (4-12) $\mu$ m y con punteaduras simples en las caras radiales.

\section{Arbutus glandulosa (lámina 2, figuras $d-f, g$ )}

Características macroscópicas. La madera presenta diferencia de color entre albura y duramen, la albura es rosa (5YR 7/4) y el duramen amarillo rojizo (7.5YR $6 / 6$ ); no tiene olor ni sabor característicos, el brillo es alto, el veteado es liso, la textura es fina y el hilo es recto. Los vasos y los radios son visibles a simple vista, los anillos de crecimiento están poco marcados.

Características microscópicas. Los anillos de crecimiento están marcados por 4 (2-5) hileras de fibras radialmente comprimidas. La madera presenta porosidad difusa, los vasos son muy numerosos, de 70 (60-80)/ $\mathrm{mm}^{2}$, la mayoría son solitarios y múltiples radiales de 2 a 6 y $2 \%$ son agrupados de 2 a 4, de contorno angular, su diámetro tangencial es moderadamente pequeño, de $51(45-80) \mu \mathrm{m}$. Los elementos de vaso son de longitud mediana, de $376(310-480) \mu \mathrm{m}$, con placas perforadas escalariformes, de 5 (1-7) barras, de paredes terminales oblicuas y punteaduras areoladas alternas de forma oval; presentan engrosamientos helicoidales. Las punteaduras de vaso-radio son redondeadas. 


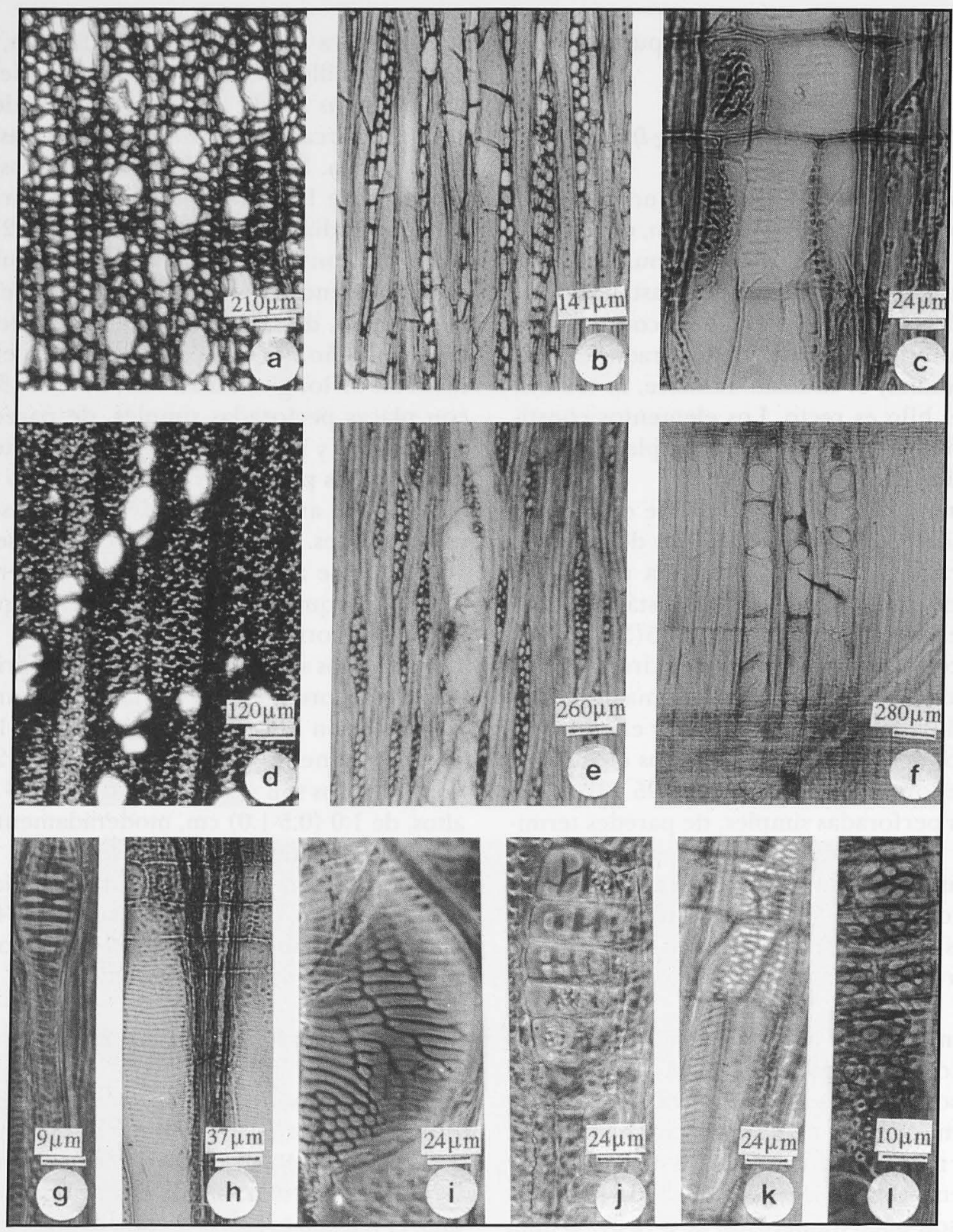

Lámina 3. Cortes transversales (izquierda), cortes tangenciales (centro) y cortes radiales (derecha); figuras a-c Styrax argenteus; $d-f$ Buddleia wrightii; figuras $g-i$ Placas perforadas; $g$ Arbutus glandulosa; $h$-i Styrax argenteus; $j-k$ Punteaduras vaso-radio; j Quercus glabrescens; k Ostrya virginiana; 1 Buddleia wrightii.

El parénquima axial es apotraqueal difuso, formado por cordones de 1 a 2 células.

Los radios son de una a cuatro series, la mayoría de tres, heterocelulares, el cuerpo está formado por células procumbentes y los extremos de 1 a 4 células cuadradas; son moderadamente numerosos, de 8 (711)/mm, extremadamente bajos, de 344 (208-804) $\mu \mathrm{m}$ y de anchura mediana, de 61 (56-72) $\mu \mathrm{m}$.

Las fibras son de tipo libriforme y fibrotraqueidas, moderadamente cortas, de 740 (510-980) $\mu \mathrm{m}$, diámetro mediano, de 35 (22-45) $\mu \mathrm{m}$, paredes delgadas, de 9 (5-10) $\mu \mathrm{m}$, algunas con punteaduras simples y otras areoladas. Presentan engrosamientos helicoidales. 
6. Arbutus tessellata (lámina 2, figuras g-i y lámina 3, figura $g$ )

Características macroscópicas. La madera presenta diferencia de color entre albura y duramen, la albura es rosa (5YR 7/4) y el duramen amarillo rojizo (7.5YR 6/6); no tiene olor ni sabor característicos, el brillo es alto, el veteado es liso, la textura es fina y el hilo es recto. Los vasos y los radios son visibles a simple vista, los anillos de crecimiento están poco marcados. Características microscópicas. Los anillos de crecimiento están marcados por 7 (4-9) hileras de fibras de madera tardía radialmente comprimidas. La madera presenta porosidad difusa, los vasos son numerosos $20(15-38) / \mathrm{mm}^{2}$, la mayoría solitarios y múltiples radiales de 2 a 7 y $2 \%$ son agrupados de 4 a 5 , de contorno angular, su diámetro tangencial es moderadamente pequeño, de 57 (40-92) $\mu \mathrm{m}$. Los elementos de vaso son de longitud mediana, de 420 (304-528) $\mu \mathrm{m}$, con placas perforadas simples y escalariformes de 3 ( 1 a 4 ) barras, de paredes terminales oblicuas y punteaduras areoladas alternas de forma ligeramente angular, presentan engrosamientos helicoidales y gomas. Las punteaduras de vaso-radio son redondeadas.

El parénquima axial es apotraqueal difuso, formado por cordones de 2 a 3 células.

Los radios son de una a tres series, la mayoría de tres, heterocelulares, el cuerpo está formado por células procumbentes y los extremos de 1 a 3 células cuadradas; son moderadamente numerosos, de 6 (49) $/ \mathrm{mm}$, extremadamente bajos, de 435 (256-744) $\mu \mathrm{m}$ y de anchura mediana, de 48 (30-60) $\mu \mathrm{m}$.

Las fibras son de tipo libriforme y fibrotraqueidas, son moderadamente cortas, de 750 (520-960) $\mu \mathrm{m}$, diámetro mediano, de 30 (24-40) $\mu \mathrm{m}$, paredes delgadas, de 9 (6-10) $\mu \mathrm{m}$, algunas con punteaduras simples y otras areoladas. Presentan engrosamientos helicoidales.

\section{Styrax argenteus (lámina 3 , figuras $a-c, h, i$ )}

Caracteristicas macroscópicas. La madera presenta diferencia de color entre albura y duramen, la albura es rosa (7.5YR 7/4) y el duramen castaño rojizo (5YR $6 / 4)$; no tiene olor ni sabor característicos, el brillo es mediano, el veteado es liso, la textura es fina y el hilo es recto. Los elementos constitutivos de la madera son visibles con lupa, los anillos de crecimiento están poco marcados.

Características microscópicas. Los anillos de crecimiento, están marcados por $3(2-5)$ hileras de fibras radialmente comprimidas. La madera presenta porosidad difusa, los vasos son moderadamente numerosos $10(14-33) / \mathrm{mm}^{2}$, solitarios, de contorno angular, su diámetro tangencial es mediano, de 104 (56-140) $\mu \mathrm{m}$. Los elementos de vaso son muy largos, de 1475 (960-2192) $\mu \mathrm{m}$, con placas perforadas escalariformes, de 38 (20 a 45) barras, algunas reticuladas y simples, sus paredes terminales son oblicuas, de puntuaciones areoladas opuestas de forma redondeada. Las punteaduras de vaso-radio son simples y redondeadas.

El parénquima axial es apotraqueal difuso, formado por cordones de 5 a 8 células.

Los radios son de una a dos series, la mayoría de dos, heterocelulares, el cuerpo está formado por células procumbentes y los extremos de 3 a 12 células cuadradas; son moderadamente numerosos, de 6 (4$10) / \mathrm{mm}$, muy bajos, de 691 (440-1600) $\mu \mathrm{m}$ y moderadamente finos, de $32(20-40) \mu \mathrm{m}$.

Las fibras son de tipo libriforme y fibrotraqueidas, muy largas, de 2259 (1240-2960) $\mu \mathrm{m}$, diámetro mediano, de 47 (30-60) $\mu \mathrm{m}$, paredes delgadas, de 9 (710) $\mu \mathrm{m}$, las primeras presentan punteaduras simples y las otras punteaduras areoladas pequeñas.

\section{Buddleia wrightii (lámina 3 , figuras $d-f, l$ )}

Características macroscópicas. La madera presenta diferencia de color entre albura y duramen, la albura es castano amarillento claro (10YR 6/4) y el duramen castaño grisáceo (10YR 5/2); no tiene olor característico y su sabor es ligeramente amargo, el brillo es mediano, el veteado es suave, la textura es mediana y el hilo es recto. Los elementos constitutivos de la madera son visibles con lupa, los anillos de crecimiento están poco marcados.

Características microscópicas. Los anillos de crecimiento están marcados por 2 (1-3) hileras de fibras radialmente comprimidas. La madera presenta porosidad difusa, los vasos son moderadamente numerosos, de $13(8-20) / \mathrm{mm}^{2}$, la mayoría son múltiples radiales de 2 a 5 y $3 \%$ son solitarios, de contorno angular, su diámetro tangencial es mediano, de 120 (72-168) $\mu \mathrm{m}$. Los elementos de vaso son moderadamente cortos, de 336 (240-456) $\mu \mathrm{m}$, con placas perforadas simples, de paredes terminales oblicuas y puntuaciones areoladas alternas de forma oval. Las punteaduras de vaso-radio son simples y angulares.

El parénquima axial es apotraqueal difuso muy escaso, formado por cordones de 2 a 3 células.

Los radios son de una a cuatro series, la mayoría de tres heterocelulares, el cuerpo está formado por células procumbentes y los extremos de 1 a 2 células cuadradas; son moderadamente numerosos, de 7 (59) $/ \mathrm{mm}$, extremadamente bajos, de 435 (264-792) $\mu \mathrm{m}$ y extremadamente finos, de 12 (8-16) $\mu \mathrm{m}$. Presentan gomas de color ámbar. 
Las fibras son de tipo libriforme, medianas, de 1228 (792-1920) $\mu \mathrm{m}$, diámetro fino, de 14 (16-28) $\mu \mathrm{m}$, paredes gruesas, de 8 (4-9) $\mu$ m y con punteaduras simples en las caras radiales.

\section{Discusión}

La madera de las especies estudiadas presenta diversidad en el color que va del blanco, rosa, castaño pálido, castaño amarillo claro, castaño grisáceo hasta castaño y amarillo rojizos; por otro lado, ninguna tiene olor ni sabor característicos, con excepción de $B$. wrightii que tiene sabor ligeramente amargo; el brillo es mediano en la mayoría y alto en las dos especies de Arbutus; el veteado es liso y la textura fina en seis especies, sólo $Q$. dysophylla y $B$. wrightii presentan veteado suave y textura mediana y todas tienen hilo recto.

Considerando estas características macroscópicas y tomando en cuenta las propiedades que Kribs (1968) y Jane (1970) analizan para los usos de la madera, es posible ubicarlas como maderas fáciles de tallar, con acabados de buena calidad, por lo que se recomiendan su usos para escultura, y torneados, donde se pongan de manifiesto sus características estéticas. Por sus colores claros, ausencia de olor y sabor, se sugiere emplearias en productos en los que estén relacionados los sentidos del gusto y del olfato, como palillos, abatelenguas y recipientes para comida; excepto B. urightii que tiene sabor ligeramente amargo.

En cuanto a las características microscópicas, una diferencia importante entre las especies estudiadas es la porosidad, ya que los dos encinos la presentan anular y las otras seis difusa.

La porosidad anular es un carácter que influye en las propiedades físicas, mecánicas y estéticas, ya que éstas dependen de la anchura de los anillos de crecimiento y por tanto de la proporción de madera temprana y tardía existente en cada uno. La madera de anillos angostos es suave, ligera, con menor cantidad de fibras, que la hace adecuada para chapa; por el contrario anillos anchos, hacen madera más dura y densa, ideal para propósitos de construcción (Jane, 1970; Panshin, 1970; Hoadley, 1989). Por otro lado, la porosidad difusa, encontrada en las otras seis especies, da cualidades constantes a la madera (Panshin, 1970). A pesar de que los encinos mexicanos presentan este carácter anatómico notable, están excluidos del manejo forestal y son considerados como plaga forestal por los manejadores del recurso (Reyes, 1994).

Otros caracteres que influyen en las propiedades de la madera y por lo tanto en su utilización son las dimensiones y número de los radios, ya que por un lado favorecen el veteado y la textura, pero también intervienen en las contracciones, provocando rajaduras que pueden bajar su calidad y, las fibras, que intervienen en la densidad de la madera (Kollmann, 1968).

De las especies estudiadas, las de Quercus presentan además de los radios uniseriados, los multiseriados que son poco numerosos. En $Q$. dysophylla son moderadamente altos y anchos, en $Q$. glabrescens son altos y moderadamente anchos. Cabe resaltar que en este último, estos elementos no son visibles a simple vista, lo que se refleja en su veteado que es liso y la textura fina, algo realmente raro en la madera de encino. Las otras especies presentan radios moderadamente finos, de una a cuatro series y extremadamente bajos $A$. acuminata, presenta además radios agregados. De acuerdo con estas características la madera de estas especies no tendría problemas de contracciones ni de secado (Ávila, 1985).

Con respecto a las fibras en $A$. acuminata son moderadamente cortas y de pared delgada; en los dos Quercus son de longitud y pared mediana, en O. virginiana y $B$. wrightii, son medianas y de pared gruesa. En los Arbutus son moderadamente cortas y $S$. argenteus las presenta muy largas ambas tienen pared delgada. En todos los casos son de diámetro mediano.

Además de la utilidad de los estudios anatómicos para sugerir usos, determinar algunas propiedades, también son importantes para la identificación de la madera, información básica para la sustitución de especies (Hoadley, 1990).

En la identificación de la madera todas las características anatómicas tienen un papel importante por lo que en estas especies se distinguen las siguientes: en $A$. acuminata la placa escalariforme, las puntuaciones vasculares opuestas y los radios uniseriados así como los agregados; en Q. virginiana los engrosamientos helicoidales; en los Quercus la porosidad anular, los poros solitarios, las traqueidas vasicéntricas y los radios uniseriados y multiseriados; en los Arbutus los engrosamientos helicoidales tanto en los elementos vasculares como en las fibras y la placa escalariforme; en $S$. argenteus los poros solitarios, la placa escalariforme ramificada, reticulada y simple así como el número de células cuadradas de los márgenes de los radios y, finalmente, en $B$. wrightii el color del duramen, los radios extremadamente finos y el tipo y forma de las puntuaciones vaso-radio.

Los resultados obtenidos se pueden emplear en los procedimientos de identificación de taxa, en la sustitución comercial de especies, en la sugerencia de algunos usos y para explicar algunas propiedades tecnológicas de la madera (Kollmann y Coté, 1968).

En cuanto a su influencia en las propiedades tecnológicas, Quintanar (datos no publicados), al me- 
dir la velocidad del sonido en las ocho especies, encontró que los Quercus presentaron valores altos, lo cual es resultado de la presencia de la porosidad anular, radios poliseriados anchos y altos y mayor cantidad de fibras, comparado con valores bajos obtenidos para las otras seis especies que presentan porosidad difusa, radios finos y bajos y menos fibras.

De todo lo anterior se recomienda que en los procesos de transformación de estas especies, se debe tener mucha precaución en el aserrío y secado de los encinos dadas las características de las dimensiones de los radios multiseriados.

Las especies dadas a conocer en este trabajo, deben destinarse a programas de mejoramiento genético y control de caracteres a través de planes silviícolas, para contar de manera constante con madera de alta calidad e introducirla al mercado y de este modo ampliar los usos a los que se destina actualmente con lo cual se puede contribuir a la explotación racional del recurso forestal.

\section{Usos}

Por las características anatómicas que presenta la madera de las especies estudiadas, se recomienda su utilización en la elaboración de productos de alta calidad, como en ebanistería, pisos, escaleras, lambrín, canceles y decoración en general, en donde se ponga de manifiesto su belleza. Asimismo por su hilo recto en la producción de chapa y artículos torneados en general. Por su color, textura y veteado en juguetes, muebles infantiles y hormas para calzado. Básicamente por su color, ausencia de olor y sabor, en diversos recipientes para alimentos sólidos o líquidos, abatelenguas y palillos (a excepción de $B$. wrightii que tiene sabor ligeramente amargo). Y por último dada la estructura homogénea que presenta se recomienda en cajas, puentes y brazos de instrumentos musicales de cuerda y muebles de instrumentos de percusión.

\section{Agradecimientos}

Las autoras agradecen a las autoridades de la Unidad de Administración Forestal núm. 2 del Municipio de Ciudad Guzmán, del estado de Jalisco, especialmente al Ing. Jaime Bocanegra Gallegos y al personal técnico del Ejido Llano Grande, del Municipio de Chignahuapan, estado de Puebla, especialmente a la Ing. Laura Cruz Medrano, las facilidades brindadas para la recolección del material de estudio. A la Biól. Lourdes Aguilar Enríquez, la identificación de las especies de Quercus; al INIFAP, el apoyo en la elaboración de las tablillas. Asimismo a los M. en C. Adol- fo Espejo, Ana Rosa López Ferrari e Irma Reyes, sus valiosos comentarios en la revisión del manuscrito y al Sr. Jorge Lodigiani el procesado de una parte del material fotográfico, los cuatro del Departamento de Biología de la Universidad Autónoma MetropolitanaIztapalapa. Este trabajo estuvo apoyado en parte por la SEP-DIGICSA (Convenio No. C90-01-0282, No. de registro 900828).

\section{Literatura Citada}

Ávila C. 1985. Secado en estufa de la madera aserrada de mezclas de encinos rojos y blancos. II Seminario nacional sobre utilización de encinos. Guadalajara, Jalisco, México.

Barajas J. 1980. Anatomía de maderas de México No. 3. Diez especies del bosque caducifolio de las cercanías de Xalapa,Ver. México. Biótica 5:23-40.

Corral G. 1981. Anatomía de la madera de siete especies del género Quercus. Boletín Técnico del Instituto Nacional de Investigaciones Forestales 72:11-53.

Chattaway M. 1932. Proposed standards for numerical values used in describing woods. Tropical Woods 29:20-28

Dalla Torre C. and Harms H. 1963. Genera siphonogamarum ad systema englerianum conscripta. Wiesbaden, Verlag fur Wissenschffliche, Neudrucke GMBH.

De la Paz Pérez-Olvera C. 1974. Anatomía de la madera de cinco especies de encinos de Durango. Boletín Técnico del Instituto Nacional de Investigaciones Forestales 43:1 1-32.

De la Paz Pérez-Olvera C. 1976. Características anatómicas de cinco encinos de México. Boletín Técnico del Instituto Nacional de Investigaciones Forestales 46:9-33.

De la Paz Pérez-Olvera C. y Corral G. 1980. Estudio anatómico de la madera de once especies de angiospermas. Boletín Técnico del Instituto Nacional de Investigaciones Forestales 64:1-79.

De la Paz Pérez-Olvera C., Carmona T. y Rogel M.A. 1980. Estudio anatómico de la madera de 43 especies tropicales. Boletín Técnico del Instituto Nacional de Investigaciones Forestales 63:235-240.

De la Paz Pérez-Olvera C. 1982. Estructura anatómica de cinco especies del género Quercus. Boletín Técnico del Instituto Nacional de Investigaciones Forestales 88:13-62.

De la Paz Pérez-Olvera C., Olvera L.P, y Corral G. 1982. Estudio anatómico de la madera de 26 especies de angiospermas de clima templado. Boletín Técnico del Instituto Nacional de Investigaciones Forestales 91:1-126.

De la Paz Pérez-Olvera C. 1985 . Variación de la estructura anatómica de los encinos y su efecto en el aprovechamiento de los mismos. II Seminario Nacional Sobre Utilización de Encinos. Publicación Especial del Instituto Nacional de Investigaciones Forestales 49:291-312.

De la Paz Pérez-Olvera C, y Quintanar A. 1994. Características anatómicas de la madera de cinco especies del estado de Jalisco, México. Acta Botánica Mexicana 27:75-87. 
Franklin G.L. 1946. A rapid method of softening wood for microtome sectioning. Tropical Woods 88:35-41.

Guridi L. 1980. La madera en las artesanías del estado de Michoacán. Boletín Divulgativo del Instituo Nacional de Investigaciones Forestales 50:1-12.

Hoadley B. 1989. Understanding wood. The Taunton Press. Connecticut. USA.

Hoadley B. 1990. Identifying wood. The Tauton Press. USA.

IAWA Committee. 1937. Standard terms of length of vessel members and wood fibers. Tropical Woods 51:21-22.

IÁWA Committee. 1939. Standard terms of size for vessel diameter and ray width. Tropical Woods 59:51-52.

IAWA Committee. 1989. IAWA list of microscopic features for hardwood identification. International Association of Wood Anatomists 10:219-332.

Jane J.W. 1970. The structure of wood. Adam \& Charles Black. London.

Johansen D.A. 1940. Plant microtechnique. McGraw-Hill. New York.

Koller A. 1927. Preparing woody tissues for making microscopic mounts. USDA. Forest. Service. Madison. 7p.

Kollmann F. and Coté W. Principles of wood science and technology I. Solid wood. Springer-Verlag. New York.

Kribs D.A. 1968. Commercial foreign woods on the American market. Dover. New York.

Munsell Color Company. 1990. Munsell soil color charts. Baltimore, Maryland.
Ortega F., Guerrero F., Carmona T. y Córdoba C. s/f. Angiospermas arbóreas de México núm. 1. Anatomía de la madera de veintiocho especies de Cosautlán de Carvajal, Veracruz. Boletín Técnico Instituto Nacional de Investigaciones sobre Recursos Bióticos 19:117-153.

Panshin A, de Zeeuw C. 1970. Textbook of wood technology. McGraw-Hill. New York.

Rebollar S. 1977. La madera de Alnus firmifolia y sus usos. Ciencia Forestal 2:51-63.

Reyes I. 1994. Los bosques de encino en México: riqueza que se convierte en carbón. Contactos 3a. época 8:28-35.

Rzedowski J. 1988. La vegetación de México. Limusa. México, D. F.

Rzedowski J. 1992. Diversidad y orígenes de la flora fanerogámica de México. Ciencias 6:47-56.

SARH. 1989. México forestal. Avances de la producción maderable. Secretaria de Agricultura y Recursos Hidráulicos. Tríptico.

Standley P. 1924. Trees and shrubs of Mexico. part 4. 23:10991130.

Standley P. and Steyermark J. 1952. Flora of Guatemala. Fieldiana Botany, part III. 24:359-369.

Standley P. 1961. Trees and shrubs of Mexico. part 1. 23: 167-169.

Tortorelli L. 1956. Maderas y bosques argentinos. ACME. Buenos Aires.

Zavala F. 1990. Los encinos mexicanos: un recurso desaprovechado. Ciencia y Desarrollo 7:43-51. 